\title{
CAPÍTULO 2 \\ DERECHO A LA CIUDAD Y CIUDADANIAS SOSTENIBLES: ENTRE LA EXIGENCIA AL ESTADO Y LA AUTO-ORGANIZACIÓN CIUDADANA
}

DOI: http://dx.doi.org/10.18616/pgt02

Julián Alberto Caicedo-Medina Jaime Hernández-García 


\section{INTRODUCCIÓN}

La ciudad Latinoamericana se debate entre la exigencia de derechos que hacen los ciudadanos al Estado, bien sea porque hacen parte de las políticas públicas, pero no se cumplen; o porque no están reglamentados, pero son fundamentales para la calidad de vida urbana. $\mathrm{Y}$, por otra parte, las distintas acciones que los ciudadanos realizan por su cuenta, a veces inclusive contraviniendo las normas. Irazábal (2008, p. 20) describe como en América Latina "Ios ciudadanos actúan por y dentro de las comunidades locales", desplazando muchas veces la responsabilidad del Estado. Este capítulo analiza esta dicotomía desde dos conceptos; por una parte, desde "el derecho a la ciudad" planteado inicialmente por Lefebvre (1969) que ha derivado en otros derechos habitacionales y territoriales, así como en perspectivas teóricas y metodológicas, como por ejemplo la justicia espacial (RAWLS, 2006; SOJA, 2009), y un concepto emergente denominado "ciudadanías sostenibles" [sustainability citizenship] (HORNE et al., 2016) que agrupa conceptos como placemaking, resiliencia, sostenibilidad y ciudadanía. El artículo discute la pertinencia teórica de estos conceptos en la búsqueda de la sostenibilidad urbana, ilustrados desde dos desarrollos urbanos y de vivienda en las periferias de Bogotá.

El derecho a la ciudad de Lefebvre (1969) se enmarca en la reflexión marxista, cuestionando las desigualdades sociales presentes en el espacio urbano, su deshumanización, y planteando la recuperación del ser humano como eje construcción de la ciudad. Lefebvre propone abordar el problema desde las transformaciones sociales como determinantes del cambio social que permitirá la auto-transformación del modo de producción del espacio y el espacio mismo. En otras palabras, el derecho a la ciudad tendrá que ser acompañado de una revolución económica planificada y auto gestionada dirigida a satisfacer las necesidades sociales, una revolución política, una revolución cultural y una revolución en la vida cotidiana. Así el derecho se plantea como algo que los ciudadanos deben 
exigir al Estado, y se vincula con la organización ciudadana, las luchas sociales y los cambios estructurales.

Por otra parte, el concepto de ciudadanías sostenibles surge también de la organización ciudadana, pero están orientadas por la acción para generar cambios por ellos mismos (HORNE et al., 2016), y se puede decir que se vincula o es una evolución del concepto de placemaking, más aplicable a contextos periféricos globales (BEZA; HERNÁNDEZ-GARCÍA, [s.d.]). Mientras que el placemaking trabaja mejor en contextos formales y con mecanismos establecidos de participación, las ciudadanías sostenibles pueden actuar en contextos informales, donde la iniciativa comunitaria es fundamental y los procesos participativos formales pueden o no estar disponibles (BEZA; HERNÁNDEZ-GARCÍA, [s.d.]). Sin embargo, la acción no es solamente referida a "actividades concretas, como votación, libertad de expresión, beneficios públicos de cualquier tipo, etc." (JELIN, 1996, p. 104); la acción, en el contexto de las ciudadanías sostenibles, se considera en términos de reunir la heterogeneidad de los actores, en un contexto específico, para pensar o identificar una cuestión que desean abordar; planificar cómo abordar esa cuestión; y establecer medidas que permitan superar el problema (BEZA, 2016a, 2016b). En este sentido, las "acciones" se posicionan como "cualidades auto-sostenibles y expansivas" (JELIN, 1996, p. 104), donde éstas generan cambios socio-espaciales que pueden contribuir a crear un ambiente sostenible (TURNER, 2012).

Dentro de este marco, este capítulo discute la pertinencia de los dos conceptos usando para ello dos ejemplos de la ciudad de Bogotá; por una parte, el macroproyecto de vivienda nueva en el sector de Soacha "Ciudad Verde", y, por otra parte, el desarrollo informal de Caracolí en el área de Ciudad Bolívar. El primer caso de basa en la investigación doctoral de uno de los autores, quien desarrolló investigación empírica y documental desde 2013 hasta la fecha, y el segundo caso se fundamenta en una indagación longitudinal de campo desde 2013 hasta la fecha y que se halla publicada parcialmente (BEZA; HERNÁNDEZ-GARCÍA, [s.d.]; HERNÁNDEZ-GARCÍA, 2016a). Después de esta introducción se presenta una elaboración mayor de los dos conceptos que rigen el desarrollo del 
capítulo, para pasar luego a discutirlos utilizando dos casos en Bogotá. La sección final de conclusiones analiza el interés y utilidad de estos conceptos a la luz de la búsqueda de la sostenibilidad urbana.

\section{MARCO TEÓRICO}

La sostenibilidad urbana como resultado de la construcción del hábitat es uno de los intereses de la sociedad, en la que se desarrollan los procesos formales e informales para su configuración. El establecimiento de ésta como objetivo consensuado es promovido por el Estado, como interés de superación de las vulnerabilidades socio territoriales y económicas que reivindican la equidad, la inclusión y la libertad de los ciudadanos. En este sentido en el presente artículo se examinan dos conceptos que orientan intervenciones en el espacio urbano y que buscan definir en el hábitat residencial bajo esquemas de gestión diferentes, los mecanismos para alcanzar la sostenibilidad urbana que beneficie a los residentes de cada intervención.

La discusión sobre el derecho a la ciudad reconoce que su materialización es la garantía del bienestar de la sociedad en virtud del acceso equitativo e inclusivo a las condiciones físicas y no físicas que lo determinan. El derecho a la ciudad y sus derechos subsidiarios se hacen concretos a través de las políticas públicas, y son los acuerdos internacionales suscritos los que permiten su adopción al ser parte del bloque de constitucional que deben regir las intervenciones del Estado. El derecho a la ciudad de Lefebvre ${ }^{(1969)}$ se enmarca en la reflexión marxista, cuestiona las desigualdades sociales presentes en el espacio urbano, su deshumanización y se plantea la recuperación del ser humano como eje de la ciudad. Además, afirma que el sentido histórico de la ciudad y su relación con los habitantes está en riesgo con la homogenización de la forma urbana propuesta en el funcionalismo del movimiento moderno en la Carta de Atenas, cuyo planeamiento urbano promoviera la especialización y segregación del espacio urbano, afectando la vitalidad y las condiciones 
de vida en la ciudad. Esta orientación agrava los problemas generados bajo el desarrollo capitalista de la ciudad, que borraba la ciudad anterior, desconociendo el valor histórico y cultural de la sociedad. Lefebvre ${ }^{(1969)}$ sugiere abordar el problema desde las transformaciones sociales como determinante del cambio social que permitirá la auto-transformación del modo de producción del espacio y el espacio mismo.

En otras palabras, el derecho a la ciudad tendrá que ser acompañado de una revolución económica planificada y auto gestionada dirigida a satisfacer las necesidades sociales, una revolución política, una revolución cultural y una revolución en la vida cotidiana. Lefebvre propone el análisis crítico al Estado y al modo de producción capitalista desde el espacio, reconociendo que la ciudad es un producto político y, a su vez, un instrumento de cambio de la sociedad (BUSQUET, 2013). Este derecho implica la transformación y configuración de la organización físico espacial a partir de la participación de los habitantes en la organización social que orienta el proceso de construcción de la ciudad, esto es en palabras de Mathivet (2010), una dinámica de transformación y conquista en la que los movimientos sociales son el motor para lograr el derecho a la ciudad.

El derecho a la ciudad es una demanda social individual y colectiva del deseo a gozar de ella como ciudadano y como comunidad de las oportunidades y servicios que ofrecen las áreas urbanas, participar en su gobernanza y habitar y usar la ciudad. En la práctica, esa consideración reconoce el goce de una ciudadanía plena de derechos, la participación en la producción y gestión social del hábitat, la gestión democrática de la ciudad, a través de la participación de la sociedad de forma directa; propugna la función social de la propiedad y de la ciudad, donde predomine el bien común sobre el derecho individual de propiedad, lo que implica el uso socialmente justo y ambientalmente sustentable del espacio urbano (SUGRANYES, 2010). La ciudad es un espacio político, donde se posibilitan las expresiones de las voluntades colectivas, es un espacio de solidaridad, pero también para el conflicto (BORJA, 2003). La construcción de la ciudad ofrece la posibilidad de configurarla como el espacio para vivir con dignidad, reconocerse como parte de ella y donde 
se garantice el acceso equitativo a los recursos -bienes y servicios- que se ofrecen en ella: empleo, salud, educación, vivienda, cultura, etc. (HARVEY, 2008a).

La urbanización se convierte en la garantía de la construcción del derecho a la ciudad como determinación de las condiciones del desarrollo de una plena ciudadanía basada en el ejercicio y goce del autogobierno, la transformación física del espacio se manifiesta en los cambios sociales de la sociedad y viceversa, el goce de las libertades y derechos al desarrollo, al hábitat y otros que avalan el bienestar individual y colectivo de la sociedad. El derecho a la ciudad se materializa a través de un conjunto de derechos que se constituyen en exigencias al Estado:

- Derecho a un hábitat, un entorno habitable que facilite el desarrollo del tejido social bajo condiciones de habitabilidad y sustentabilidad adecuados que permitan la condición de habitar dignamente, desarrollarse plenamente como individuo e insertarse como miembro de la comunidad en un espacio socio físico acorde a sus necesidades socioculturales, políticas, económicas y geográficas para el desarrollo humano en un ambiente sano y seguro, con acceso al agua, los servicios públicos y a la vivienda (DEDE DELFINO, 2008; GLEDHILL, 2010; TORRES TOVAR, 2009).

- Derecho a ser parte de la ciudad (sentido de cohesión social y construcción colectiva), a través del ejercicio de la ciudadanía, evitando todo tipo de discriminación negativa, brindando especial protección a los grupos y personas en situación de vulnerabilidad económica, étnica y social, y garantizando mecanismos de integración inclusión y equidad para todos los habitantes (CIUFFOLINI, 2008; PONCE SOLÉ, 2015).

- Derecho a la convivencia, bajo un marco jurídico y social que garantice la seguridad pública y la convivencia pacífica, solidaria y multicultural (CRUZ RODRÍGUEZ, 2013). 
- Derecho al gobierno a la ciudad, como participantes activos en la construcción social e institucional del hábitat, articulada a la planificación de la ciudad, que brinde impulso de la economía solidaria y políticas impositivas progresivas (FLORIÁN BORBÓN, 2002; MATHIVET, 2009).

- Derecho a la igualdad de derechos, como espacio social donde se garantice la libertad e integridad en la participación política, la justicia en el acceso y permanencia en los diferentes aspectos de la vida cotidiana, así como en el acceso a los servicios urbanos como salud, educación y empleo (COPEVI, 2005; MATHIVET, 2010).

- Derecho a vivir dignamente, en un espacio que posibilite la construcción social del hábitat, bajo condiciones de desarrollo del espacio urbano equitativo, inclusivo y sustentable (MURILLO et al., 2010; SUGRANYES; MATHIVET, 2010).

Por su parte, las Ciudadanías Sostenibles (sustainability citizenship) es un término acuñado en el libro editado por Horne y otros (2016), que se relaciona con placemaking (PIERCE; MARTIN; MURPHY, 2011), resiliencia (COAFFEE; LEE, 2016), sostenibilidad (BULKELEY, H.; BETSILL, 2003) y ciudadanía (TURNER, 2012). Nelson (NELSON, 2016, pp. 17-18)lo define como un nuevo concepto de ciudadanía que tiene 5 propiedades:

- el medio ambiente y su cuidado son centrales en cualquier argumento relacionado con lo urbano,

- es una perspectiva local y global al mismo tiempo, alterando el marco contractual estatal-individual de muchos discursos tradicionales de ciudadanía,

- se apoya en responsabilidades colectivas asociadas con los valores de solidaridad, seguridad y sostenibilidad, 
- se caracteriza por formas horizontales de gobernanza compartida (como las relaciones entre iguales y las estructuras de trabajo en red), y

- su énfasis está en "ser" y "hacer" más que en "tener".

El concepto puede entenderse también como una "ciudadanía urbana de sostenibilidad" que "sitúa a los ciudadanos como agentes de cambio social que impulsan a crear entornos urbanos, entornos y estilos de vida que son comunitarios y ambientalmente sostenibles" (HORNE et al., 2016, p. 4). Por lo tanto, para el desarrollo de ciudad y la transformación socio-espacial de la misma, las ciudadanías sostenibles pueden contribuir a la creación entornos ambiental y socialmente más sostenibles. Sin embargo, la aplicación de este concepto puede desafiar el proceso de planificación "formal" y establecido usualmente para la creación y transformación de los lugares; ya que se fundamenta en la autoorganización, la auto-gestión y la acción, pudiendo obtener resultados fuera del proceso de planificación "formal".

Las ciudadanías sostenibles se basan en una ciudadanía activa y comprometida para construir la agencia en el proceso de desarrollo urbano y de gobernanza (BEZA, 2016a, p. 141); independientemente de su contexto espacial y el resultado, es decir, donde el proceso y las motivaciones de él, son más importantes. Y aunque, los debates actuales sobre la creación de lugares incluyen cuestiones de gestión (es decir, el mantenimiento de los lugares) el tema de la gobernanza es en gran medida ausente. Se supone que, debido a la creación de lugares en torno a la planificación "formal" y los entornos urbanos públicos, una vez que se haya realizado algo se mantendrá y se proporcionará la prestación de servicios por parte de una autoridad responsable. Esto no es siempre el caso, y si la ciudadanía no está comprometida, muchas veces mantener esas intervenciones se hace imposible.

De acuerdo a lo anterior, por una parte la orientación a la acción que puede desafiar (o reemplazarla por inexistente) la planificación formal, y por otra parte la necesidad de involucrar a todos los interesados de un 
entorno particular; es que el concepto de ciudadanías sostenibles puede ser muy útil en ambientes urbanos donde la autoridad municipal sea mínima o inexistente, debiendo entonces los residentes auto-organizarse y hacerse cargo de su entorno urbano, de servicios y de vivienda (ver HERNÁNDEZGARCÍA, 2016b). Este es el caso en gran medida, de los sectores informales urbanos en América Latina, y concretamente en Colombia. Los barrios informales o populares de Bogotá se crean a través de individuos que se unen por necesidad, por opción, o más frecuentemente por ambas razones- para crear o transformar su entorno urbano y de vivienda. En este sentido, los individuos se empoderan para promover cambios en su entorno urbano. $Y$ aunque su agenda no puede ser considerada como un movimiento social, actúa como uno (HERNÁNDEZ-GARCÍA, 2012) y apoya la realización de resultados relacionados con el público. Lo que resulta, en términos de este ejemplo de trabajo en equipo, es lo que describe Butcher (2016, pp. 96-97) como:

- Un fondo colectivo de capital social del que la gente puede sacar para tomar responsabilidad y actuar,

- Motivación que va más allá del resultado mismo, y se conecta explícita o implícitamente con otros objetivos (en este caso, con el de construcción de comunidad, con la sostenibilidad),

- Estar allí uno para el otro cuando sea necesario, y

- Protocolos que son desarrollados y seguidos por todos (aunque a veces puede ser de manera inconsciente).

En este marco, las ciudadanías sostenibles pueden definirse como como una ciudadanía orientada a la acción, mediante mecanismos de auto-organización y auto-gestión, pudiendo en ocasiones desafiar la planeación formal y las normas. De manera consciente algunas veces, pero otras veces no, buscan la creación y transformación de lugares más acordes a sus propias expectativas y necesidades, muchas veces coincidiendo también con lugares más amables, y más sostenibles en lo social y en lo ambiental (SOTO VILLAGRÁN, 2012) 


\section{DISCUSIÓN}

Los procesos para la configuración de la sostenibilidad urbana se abordan desde dos perspectivas de la producción del hábitat urbano y residencial, una como resultado de un proceso formal guiado por la política de vivienda social, caso de los conjuntos residenciales del macroproyecto Ciudad Verde en Soacha, colindante al sur de la localidad de Bosa en Bogotá; y la otra como un proceso de autogestión de vivienda y espacio urbano, el caso del barrio Caracolí en la localidad de Ciudad Bolívar en Bogotá. Es decir, que en el primero se considera como un proceso social asumido por los residentes de Ciudad Verde resultante de la materialización de los elementos y atributos del hábitat producido bajo la lógica de la política bajo las orientaciones del bienestar y del goce del derecho a la ciudad. Mientras que, en el segundo, el proceso social es realizado a través de los procesos cotidianos de transformación de la vivienda y su entorno por medio de las organizaciones sociales que se constituyen para la atención de las necesidades colectivas de la comunidad residente en el barrio Caracolí. En cada proceso la situación resultante es una definición de sostenibilidad que se manifiesta en las relaciones socioeconómicas y culturales para la superación de las vulnerabilidades socio territoriales a las que se enfrentan los residentes en el proceso de habitar estos territorios. Cabe reconocer que la construcción de la sostenibilidad urbana como objetivo de la sociedad consagrado en la imagen construida, es consensuada y validada por el Estado en la legislación y las políticas públicas en materia del hábitat y sus correlatos en bienestar social, superación de la pobreza y las vulnerabilidades socioespaciales, entre otras.

\section{Macroproyecto habitacional Ciudad Verde, Soacha}

Ciudad Verde es un macroproyecto de vivienda de origen formal situado en la periferia en la periferia de Bogotá, en el sector de Soacha. El 
macroproyecto es una operación inmobiliaria de gran escala iniciada en el año 2009 en el marco de la política de vivienda basada en el modelo de gestión orientado al con subsidios (CAICEDO-MEDINA, 2006), y se desarrolla en un área aproximada de 328 hectáreas de las cuales 132 se utilizarán para a la construcción de 49.500 viviendas de Interés Social (viviendas de interés social [VIS] y viviendas de interés prioritario [VIP], para albergar a casi 200.000 personas; junto con otros usos complementarios como un proyecto urbano integral. Además, se contempla la extensión de redes viales de integración regional, urbana, zonal y local, al cual se destinan 76 hectáreas para malla vial principal, local, ciclo-rutas y transporte público. Esta zona de la ciudad continúa atrayendo población y su área urbana ha superado sus límites territoriales y administrativos.

La comprensión del derecho a la ciudad está ligada a la forma de su gestión y producción, en el sentido de no generar exclusión y permitir la participación en la gobernanza de la ciudad (BENGTSSON, 2001) y el acceso a la vivienda. Bajo el modelo de gestión y producción habitacional se considera la participación de los promotores privados liderando la gestión urbana, se desarrolla en proyectos para diversos sectores como el desarrollo de planes de renovación urbana, nuevas intervenciones que consideran usos industriales, comerciales, servicios y vivienda para los diferentes estratos socioeconómicos.

El proyecto tiene como objetivo "la habilitación de suelo apto para el desarrollo de proyectos de vivienda, especialmente de vivienda de interés social (VIS) y vivienda de interés prioritario (VIP), y otros usos, dentro de un proyecto integral que contempla la dotación de servicios públicos domiciliarios, la extensión de redes viales de integración regional, urbana, zonal y local, la creación de espacios públicos y la generación de suelo para equipamientos colectivos; desarrollando asentamientos urbanos con altos parámetros de calidad ambiental y urbanística, dentro del ámbito de aplicación de Soacha, Bogotá D.C., y Cundinamarca." -Res. 1687 - (MINISTERIO DE AMBIENTE VIVIENDA Y DESARROLLO TERRITORIAL, 2009). 
La captura que el neoliberalismo ha hecho de la gobernanza urbana su herramienta de control reconfigurando los mecanismos de la gestión urbana promovida por los capitales inmobiliarios globales, imponiendo la participación de agentes económicos que promueven el desarrollo de operaciones especulativas con el fin de tomar el beneficio a su servicio y manteniendo condiciones privilegiadas para facilitar la acumulación del capital (HARVEY, 2008b; MUÑOZ, 2008). En estos procesos se han acentuado las desigualdades sociales, culturales y políticas de los ciudadanos; las franjas de inclusión y exclusión se profundizan generando güettos que segmentan la convivencia social y la vida cotidiana (ATISBA, 2010; RODRÍGUEZ; SUGRANYES, 2011; VIERA, 2012), dejando a la población de bajos ingresos imposibilitada de satisfacer las necesidades y derechos elementales que garanticen la dignidad humana.

Para los ciudadanos la reivindicación del derecho a la vivienda y al hábitat es una demanda actual válida para reclamar el disfrute de una ciudad y no debe ser parte del discurso oficial que apacigüe el proceso de reivindicación de la inclusión social y transformación de la sociedad, en al menos los términos en los que Lefebvre ${ }^{(1969)}$ y Harvey $(2008 a, 2008 c)$ lo definen. En el marco de éste, el derecho a la vivienda digna emerge, dadas las implicaciones de este último en la construcción del espacio urbano y en la garantía del goce individual y colectivo de los derechos relacionados con asentarse en la ciudad. La vivienda como derecho, no sólo es un espacio de satisfacción de bienestar físico sino como determinante del desarrollo de las interacciones sociales, políticas, económicas y culturales (SCHÜTZ, 1996). El interés de esta demanda es luchar contra la estigmatización de los grupos en desventaja socioeconómica y minorías étnicas, contra las desigualdades y la precariedad económica. En ese sentido, el derecho a la ciudad es el espacio de reivindicación política y social por pertenecer y disfrutar la ciudad como espacio que posibilita el desarrollo y la libertad que ésta ofrece.

Los atributos que definen el derecho a la vivienda se relacionan con la definición de las características físicas que soportan la estructura social referida a la condición de habitar plenamente como ciudadano sujeto 
de derechos, bajo la comprensión del derecho a la ciudad propuesta por Lefebvre ${ }^{(1969)}$ como demanda de pertenencia a ella y como posibilidad de auto-transformación individual y colectiva como lo expresa Harvey (2007, 2008a, 2012). La interlocución para la exigencia y garantías de estos atributos es mediada por los mecanismos de administración y organización que representan a los residentes y son interlocutores válidos frente a los agentes responsables del cumplimiento de los atributos o los elementos constituyentes de estos en los términos de la atención de las demandas sociales del hábitat. La intención de proveer mejores condiciones de vida libre de los riesgos socioambientales en el macro proyecto estableciendo a una comunidad organizada a través de los conjuntos residenciales en la administración de los conjuntos y en la organización social "Agrupación Ciudad Verde", que acoge la interlocución del Macropoyecto frente a las organizaciones estatales y privadas que ofrecen servicios públicos, económicos y culturales necesarios para el bienestar de los residentes. Estas organizaciones son producto de la tipología de organización física de las viviendas acorde con el estatuto legal que esta les impone a los habitantes.

La vivienda es crucial para la creación del sentido de pertenencia a la ciudad y para el mismo concepto de lugar. No es una afirmación excesiva decir que ser privado del acceso a una vivienda adecuada es estar privado de la posibilidad misma de ser parte de y para disfrutar de la vida de la ciudad (ROLNIK; DAPENA, 2014). El derecho a la vivienda digna debería ser interpretado en su sentido más amplio como derecho a vivir en la "seguridad y dignidad", y no reducirse únicamente a la "vivienda adecuada", puesto que está inextricablemente ligado a otros derechos humanos fundamentales (GLEDHILL, 2010), entre los que se destacan el derecho al ambiente sano, a la libertad, al trabajo, a la cultura y a la identidad, entre otros. Este derecho no está limitado a la propiedad, sino que tiene implicaciones más significativas con relación a las potencialidades de desarrollo y el goce de derechos individuales y colectivos. Por lo tanto, el derecho a una vivienda adecuada ha de ser entendido como una puerta de entrada a otros derechos es una condición 
que tiene que cumplirse con el fin de garantizar el ejercicio de pertenencia en todos sus aspectos (ROLNIK; DAPENA, 2014).

En el proceso de la estructuración del hábitat residencial, a la escala del macroproyecto Ciudad Verde, desde la perspectiva de la planificación urbana esta soportada en la formalización del proceso de urbanización liderado por los promotores privados generando un sector de ciudad configurado con los servicios públicos y urbanos- y equipamientos que considera el modelo urbano según la normativa planteada y adoptada para el macroproyecto. La disponibilidad de servicios públicos y urbano es uno de los atractivos de la vivienda formal. Este proceso se inserta en la lógica subyacente en la política habitacional que busca replicar el modelo "ideal" de ciudad del discurso del derecho a la ciudad. La exigencia sobre ese modelo replicado de ciudad frente al goce del derecho se establece frente a las organizaciones sociales y administrativas válidas para la interlocución de las demandas de las condiciones de habitar de las comunidades residentes y los agentes que participan en la gestión y promoción del macroproyecto y el Estado.

La situación de exigencia de los residentes del derecho a la ciudad pasa desde apropiación y comprensión desde la situación de cada individuo, considerando los aspectos socioculturales, económicos y su experiencia de habitar la ciudad; y como a través de los mecanismos sociales establecidos en las formas de administración de los conjuntos éstos se organizan para reivindicar las demandas por el derecho a la ciudad en términos de bienestar, participación, autogobierno y sostenibilidad en el contexto del macropoyecto Ciudad Verde.

\section{Barrio Caracolí, Ciudad Bolívar}

Caracolí es un barrio de origen informal situado en la periferia sur de Bogotá, en Ciudad Bolívar. Caracolí creció a partir de urbanizadores ilegales que vendieron a los primeros ocupantes lotes sin servicios ni papeles de propiedad en los años 1990s. El barrio se ubica en una zona 
de fuerte pendiente topográfica, y datos no oficiales estiman la población en aproximadamente 5.000 personas. El barrio se legalizó en el año 2007, y los lotes individuales están aún en proceso de legalización. A la fecha el barrio tiene un nivel medio de consolidación, pero con áreas aun carentes de servicios urbanos y las mínimas calidades urbanas. Como es común en los barrios informales de Bogotá, gran parte de la fábrica urbana y espacial ha sido producto de la iniciativa y posibilidades de sus pobladores.

El concepto de ciudadanías sostenibles puede ayudar a explicar el proceso de desarrollo socio-espacial de Caracolí. La creación del entorno urbano inicia a partir de un grupo de individuos que se auto-organizaron y con la intermediación de un urbanizador ilegal, plasmaron las primeras ideas del asentamiento. Nótese como no solamente desafiaron la planeación formal, sino también infringieron las leyes; pero buscando solventar una necesidad que el Estado no era capaz de solucionar. Siguiendo la visión, las iniciativas individuales lentamente dieron paso a las acciones colectivas, a través de organizaciones formalmente establecidas, como lo es la Junta de Acción Comunal (JAC); quien canalizó (y lo sigue haciendo) ?no sin dificultades y conflictos- la participación de la comunidad y la gestión ante las entidades públicas, la transformación física del espacio urbano, impactando también lo social. Además, la heterogeneidad de los individuos implicados en la transformación de Caracolí superó de alguna manera las consideraciones de "diversidad justa" de Iveson y Fincher (IVESON; FINCHER, 2015) para producir un ambiente altamente considerado por sus residentes y el municipio.

Los espacios creados en Caracolí, sin embargo, son en gran parte experimentados sólo por sus residentes $y$, en este sentido, demarcan fronteras claras del entorno urbano y lo distinguen de otros entornos adyacentes en la localidad de Ciudad Bolívar. El resultado de estas fronteras, aunque en gran medida indistinguibles a simple vista, apoyan el argumento de Lefebvre (1997, p. 73) que el espacio social (por ejemplo, el parque principal del barrio) es un producto "de una secuencia y un conjunto de operaciones, y no puede reducirse al rango de un simple objeto [o producto]". En el caso de Caracolí, la acción colectiva contribuye 
a la unicidad de este espacio, en relación con cualquier otro de la localidad. Es importante destacar que esta diferencia establecida también apoya la discusión de Lefebvre (1997, p. 84)que argumenta que el espacio "no es a priori"; que es el resultado de un proceso de pensamiento, de experiencia y de percepción (DEWEY, 1981). En Caracolí la producción del espacio es a través de un proceso de auto-organización que transforma un entorno "vacío" que ahora tiene tanto valor como sentido para sus habitantes y el municipio. Además, esta transformación también "re - inventar" el barrio como un espacio reconocido que Miraftab (2004, p. 1) sostiene que puede ayudar a "confrontar directamente [...] a las autoridades y al status quo".

Estos procesos aquí discutidos, usan el argumento de las ciudadanías sostenibles para explorar la producción de espacio; pero no deben considerarse como una manera de liberar o relevar al Estado (en este caso al municipio) de su responsabilidad de contribuir al desarrollo de la comunidad. Por lo tanto, la acción ciudadana para la transformación espacial (IRAZÁBAL, 2008, p. 20) puede ser utilizada como medio para responsabilizar a los gobiernos y obligarlos a actuar; como por en el ejemplo de Caracolí, donde la acción de la JAC ha propiciado también algunas intervenciones del municipio en el mejoramiento del barrio. La acción del gobierno, a este respecto, sigue la dirección de la ciudadanía; lo que apoya la sugerencia de Jelin (1996) de que los aportes de la comunidad se relacionan con lo que se recibe a cambio.

Otro punto a destacar asociado a las ciudadanías sostenibles es la gobernanza. En este sentido, Caracolí es un entorno urbano establecido con una ciudadanía ya existente que ha demostrado claramente que puede actuar. Sin embargo, muchos de los espacios públicos de este entorno y los emplazamientos dentro de ellos necesitan de mantenimiento regular, y este mantenimiento debe ser responsabilidad principal del municipio. Si la comunidad lo hace, puede verse como una manera de respaldar el status quo; lo cual no necesariamente es un elemento positivo en los argumentos del desarrollo del espacio o de las ciudadanías sostenibles. En este sentido las ciudadanías sostenibles implican un modelo de gobernanza "compartido", que se bien la comunidad actúa, el Estado no puede sustraerse de su responsabilidad. 
Las ciudadanías sostenibles contribuyen a lograr resultados (BUTCHER, 2016), no solo en términos físico-espaciales, sino también sociales, ambientales y de empoderamiento político. Este concepto "abarca la responsabilidad colectiva y la acción en la transformación socio-ambiental de las ciudades" (HORNE et al., 2016, p. 1) y se logra mediante el "hacer". La acción toma forma en la participación, con "debates y actividades sobre temas de sostenibilidad" (WALS; LENGLET, 2016, p. 57) para desafiar y maniobrar alrededor de marcos establecidos; y logrando individuos actuantes y comprometidos (BUTCHER, 2016; WALS; LENGLET, 2016) En este ejemplo de "hacer" en Caracolí, "la agencia de los individuos [es decir, miembros de la comunidad] gradualmente da paso a la agencia de grupos organizados $y$, en el proceso, las organizaciones comunitarias inmaduras se hacen más fuertes" (HERNÁNDEZ-GARCíA, 2016a) y poco a poco van madurando y se van empoderando. Esta "evolución" se halla enmarcada dentro de un sistema de sostenibilidad "enfatizando" el "ser"más que el "tener". Y aunque no siempre de manera explícita, hay un trasfondo de cuidado de los recursos, de solidaridad y de ayuda mutua. Y un aprendizaje colectivo de acción y de experiencia, en la búsqueda de un compromiso socio-espacial (HORNE et al., 2016)

Sin embargo, antes de que se pueda desarrollar un pleno trabajo de sostenibilidad colectiva, los individuos en un entorno como en Caracolí, deben reunirse o estar unidos para actuar. En este sentido y en términos de espacio público en los asentamientos informales de Bogotá, los residentes son instados a actuar, no solamente debido a la necesidad, sino también a la expectativa. Por ejemplo, Irazábal (2008, p. 11) describe que en América Latina las personas se toman las calles en respuesta a preocupaciones internacionales y/o domésticas. La protesta asociada puede terminar entonces, en algún lugar, por ejemplo, una plaza existente u otro espacio público. En su ejemplo, describe como por ejemplo las personas actúan en referencia a rechazar "condiciones económicas opresivas" del neoliberalismo. Esta acción sirve de catalizador para la visión "de proyectos sociopolíticos alternativos" (ídem: 11), las personas se reúnen en reacción a "algo"; mientras que en Caracolí, por ejemplo, la unión de los individuos "implica un sentido de pertenencia" 
(BOKSER MISSES-LIWERANT, 2013, p. 348) donde los residentes se unen "para afrontar los límites existentes por una agenda política que los invisibiliza". Por lo que en la participación y confrontación se empoderan como ciudadanos del barrio, y de la ciudad. Es la demostración de "responsabilidad hacia los demás y hacia el lugar" (JELIN, 1996, p. 109), de un compromiso cívico centrado en la participación activa en la vida pública; así como en los aspectos simbólicos y éticos que confieren un sentido de identidad y pertenencia, un sentido de comunidad" (JELIN, 1996, p. 106).

Caracolí demuestra un proceso de auto-organización y autogestión que se utiliza para lograr resultados socio-espaciales. Este barrio de origen informal ofrece un contexto interesante desde el cual explorar el concepto de ciudadanías sostenibles en la creación y transformación de sus espacios urbanos principalmente. En el proceso también se construye valor y significado, que se crean a través de las experiencias participativas y en el proyecto sociopolítico, que es asumido implícita y explícitamente por los miembros del barrio y sus organizaciones sociales, en especial, la Junta de Acción Comunal.

\section{CONSIDERACIONES FINALES}

El capítulo discute la pertinencia teórica de dos conceptos en la búsqueda de la sostenibilidad urbana, ilustrados desde dos desarrollos urbanos y de vivienda en las periferias de Bogotá. Por una parte, el derecho a la ciudad, como exigencia al Estado en la provisión de servicios socio-espaciales para una vida digna y justa para los habitantes de la ciudad. Por otra, las ciudadanías sostenibles, como una apuesta ciudadana y comunitaria a la acción, al auto-desarrollo, en búsqueda de lograr para sí unas mejores condiciones de vida urbana. El derecho a la ciudad no excluye la acción ciudadana, de la misma forma que las ciudadanías sostenibles no liberan o reemplazan la responsabilidad del Estado. Se podría decir que, ambas buscan lo mismo: el mejoramiento de 
la calidad urbana, una vida digna, y unas justas condiciones para todos; y aunque la forma de aproximarse es distinta, es también complementaria. Como es también complementaria, su concepción histórica y filosófica, mientras que el derecho a la ciudad parte que la ciudad fue capturada por los intereses del capital y así dejó de pertenecer a la gente, por lo tanto, Lefebvre (1969) aboga por "rescatar el hombre como elemento principal, protagonista de la ciudad que él mismo ha construido"; las ciudadanías sostenibles parten de la responsabilidad de las personas sobre su espacio (privado, urbano y global), reivindicando una “... acción colectiva, que puede enfrentar directamente a las autoridades y el statu quo" (MIRAFTAB, 2004, p. 1), como a sí mismas. El derecho a la ciudad es un concepto diríamos que clásico y tradicional, no por lo cual ha perdido valor, quizás todo lo contario; mientras que el concepto de ciudadanías sostenibles es nuevo, es emergente, no por lo cual es demasiado joven como para ignorarlo.

En la búsqueda de la garantía de las condiciones de supervivencia digna, el derecho a la ciudad definido por Lefebvre ${ }^{(1969)}$, como el derecho a la vida urbana, transformada, renovada y no puede concebirse como el simple derecho de visita o de regreso a las ciudades tradicionales. La apuesta del Estado en la instrumentación de las políticas habitaciones se orienta en la construcción de competencias ciudadanas bajo esta lógica como una respuesta de generación de las condiciones del bienestar de la sociedad, la superación de las vulnerabilidades socioeconómicas y territoriales. Las competencias son el resultado de prácticas que manifiestan el sentido de la libertad en las decisiones individuales para la determinación del bienestar en el medio social que ofrece oportunidades físicas y espaciales para el desarrollo de la vida urbana en sus diversos ámbitos, como económico, social, cultural, político para mencionar sólo algunos.

Sin embargo, los mecanismos de operatividad de este derecho mediante la producción de la vivienda social, han sido transferidos a los agentes económicos especializados, bajo el modelo de gestión habitacionales orientado al mercado (CAICEDO-MEDINA, 2006), 
respondiendo a los intereses económicos de propios de la gestión privada; que es claramente lo que se observa en el macroproyecto Ciudad Verde. Es decir que, aunque las intenciones son buenas, no logran materializarse adecuadamente ya que los intereses de los privados no están necesariamente alineados con los del derecho a la ciudad, dada su naturaleza de obtener réditos económicos importantes. El derecho a la ciudad, es entonces restaurar el sentido de ciudad, instaurar la posibilidad del "buen vivir" para todos, y hacer de la ciudad "el escenario de encuentro para la construcción de la vida colectiva" (MATHIVET, 2010); sin embargo en la práctica, y por el uso de "intermediarios", esto no se cumple, o al menos, no se cumple totalmente.

Las ciudadanías sostenibles pueden trabajar en una variedad de entornos y circunstancias. Pueden trabajar en el espacio vacío (véase SARTRE, 2008); que es el espacio considerado como inexistente o devaluado - como en el contexto de los asentamientos informales de Bogotá -, como en Caracolí, en un ambiente donde la gente claramente tiene menos recursos que otros sectores de la ciudad. Aquí la aplicación del concepto es central, ya que las personas se auto-organizan y asumen la responsabilidad colectiva de generar y sostener los resultados urbanos que apoyan el mejoramiento de sus residentes (HERNÁNDEZ-GARCÍA, 2016a). Pero esto no puede dejarse solo, no puede pensarse que es la liberación de responsabilidades por parte del Estado, y es aquí donde el derecho a la ciudad cobra una gran importancia, ya que se trata de "hacer" de manera colectiva, Estado y comunidad, y no solo ésta última.

Los términos "membresía, identidad, pertenencia, responsabilidad y comunidad" utilizados en las ciudadanías sostenibles sugieren que los ciudadanos del barrio están interconectados y, a través de la auto-organización, pueden abordar la heterogeneidad de los actores del asentamiento informal. Es decir, la variedad de actores en el barrio se puede explicar cuando se auto-organizan. Contrario a la autoorganización es la planeación estatal en contextos formales, en los cuales no necesariamente se puede dar cuenta de la heterogeneidad o pluralidad de los miembros de la comunidad en la toma de decisiones; inclusive son 
invisibilizados en la mayoría de las ocasiones. Los lugares urbanos se crean a partir de la gente, no de las normas; puede decirse que, a partir de las relaciones y dinámicas informales, y no de las estructuras formales.

A pesar de lo anterior, la creación de lugares urbanos requiere de la interacción con el Estado gobernante formal para lograr resultados cívicos relacionados. Pero lo que falta del enfoque impulsado por la autoorganización de la gente es la consideración o deliberación de asuntos comunitarios de "orden superior"; que Iveson y Fincher $(2015$, p. 47) denominan "diversidad justa", en relación con "el interés público. Es decir, el derecho a la ciudad.

\section{REFERENCIAS}

ATISBA. Estudio Guetos en Chile. Santiago: Atisba Estudios y Proyectos Urbanos Ltda., 2010.

BENGTSSON, B. Housing as a Social Right: Implications for Welfare State Theory. Scandinavian Political Studies, v. 24, n. 4, p. 255, 2001.

BEZA, B. B. Places for sustainability citizenship. En: Sustainability Citizenship in Cities: Theory and practice. London - New York: Earthscan, 2016a. p. 139-149.

BEZA, B. B. The role of deliberative planning in translating best practice into good practice: from placeless-ness to placemaking. Planning Theory and Practice, v. 17, n. 2, p. 244-263, 2016 b.

BEZA, B. B.; HERNÁNDEZ-GARCÍA, J. From Placemaking to Sustainability Citizenship: An evolution in the understanding of community realised public spaces in Bogotá's informal settlements. [En Prensa]. Journal of Place Management and Development. Special Issue: Participatory placemaking: Concepts methods and practices, [s.d.]. 
BOKSER MISSES-LIWERANT, J. Being National/Being Transnational: Snapshots of Belonging and Citizenship. En: SNZAJDER, M.; RONIGER, L.; FORMENT, C. (Eds.). . Shifting Frontiers of Citizenship: The Latin American Experience. Leiden - London: Brill, 2013. p. 343-365.

BORJA, J. La ciudad conquistada. Barcelona: Alianza Editores, 2003.

BULKELEY, H.; BETSILL, M. Cities and Climate Change: Urban Sustainability and Global Environmental Governance. London - New York: Routledge, 2003.

BUSQUET, G. L'espace politique chez Henri Lefebvre : l'idéologie et l'utopie / Political Space in the Work of Henri Lefebvre: Ideology and Utopia. justice spatiale | spatial justice, p. 1-12, 2013.

BUTCHER, T. Co-working communities: Sustainability citizenship at work. En: Sustainability Citizenship in Cities: Theory and practice. London New York: Routledge, 2016. p. 93-103.

CAICEDO-MEDINA, J. A. Configuración y evolución del modelo de gestión habitacional en Chile y Colombia. Bitácora Urbano Territorial, v. 1, n. 1, p. 158-177, 2006.

CIUFFOLINI, M. A. El derecho a la y en la ciudad: Un estudio socio-político sobre el Programa «Mi casa, mi vida» en la Ciudad de Córdoba. Debates latinoamericanos, v. 9, n. 16, 2008.

COAFFEE, J.; LEE, P. Urban resilience: planning for risk, crisis and uncertainty. London: Palgrave, 2016. 
COPEVI. Derecho a la vivienda en el marco de los derechos humanos. México D. F.: Centro Operacional de Vivienda y Poblamiento A. C., 2005.

CRUZ RODRÍGUEZ, L. La política de vivienda en el distrito federal con perspectiva de derechos humanos cun intento fallido? Congreso Nacional de Vivienda 2013 Eje tematico 7 Suelo Urbano y Derecho a la vivienda. Anais...México, D. F.: UNAM, 2013.

DEDE DELFINO, G. Políticas públicas, derechos humanos y el acceso a la vivienda digna. Aportes Andinos, n. 21, p. 13, 2008.

DEWEY, J. The philosophy of John Dewey. (Vols. 1-2). Chicago: University of Chicago Press, 1981.

FLORIÁN BORBÓN, A. Algunas reflexiones sobre el derecho a un lugar para vivir y para evolucionar de habitantes a ciudadanos. Bogotá: Fedevivienda, 2002.

GLEDHILL, J. El derecho a una vivienda. Revista de Antropología Social, v. 19, n. 1, p. 103-129, 2010.

HARVEY, D. Breve historia del neoliberalismo. 2005. ed. Madrid: Ediciones Akal, 2007.

HARVEY, D. El neoliberalismo como destrucción creativa. The Annals of the American Academy of Political and Social Science, p. 24, 2008a.

HARVEY, D. La libertad de la ciudad. Antipoda. Revista de Antropología y Arqueología, v. 7, p. 15-28, 2008b.

HARVEY, D. El derecho a la ciudad. New left review, n. 53, p. 23-39, 2008c. 
HARVEY, D. Rebel cities: from the right to the city to the urban revolution. London - New york: Verso, 2012.

HERNÁNDEZ-GARCÍA, J. Espacios Públicos en Barrios Populares. Producción y Uso, entre lo Público y lo Privado. México: Infonavit Redalyc, 2012.

HERNÁNDEZ-GARCÍA, J. Informal settlements: Sustainability citizenship in action. En: Sustainability Citizenship in Cities: Theory and practice. [s.I.] Earthscan, 2016a. p. 129-138.

HERNÁNDEZ-GARCÍA, J. Hábitat popular, un modo alternativo de producción de espacio para América Latina? En: HERNÁNDEZ-GARCÍA, I. (Ed.). . Estética de los mundos posibles: inmersión en la vida artificial, las artes y las prácticas urbanas. Bogotá: Editorial Pontificia Universidad Javeriana, 2016b. p. 245-258.

HORNE, R. et al. Sustainability, citizenship and cities : theory and practice. London - New York: Earthscan, 2016.

IRAZÁBAL, C. Citizenship, democracy, and public space in Latin America. En: Ordinary Places, Extraordinary Events: Citizenship, Democracy and Public Space in Latin America. London - New York: Routledge (Tyoly and Francis Group), 2008. p. 11-34.

IVESON, K.; FINCHER, R. The Public City and Diversity: Rethinking the 'Public Interest'. En: GLEESON, B.; BEZA, B. B. (Eds.). . The Public City: Essays in Honour of Paul Mees. Melbourne: Melbourne University Press, 2015. p. 42-53. 
JELIN, E. Citizenship Revisited: Solidarity, Responsibility, and Rights. En: JELIN, E. ; H. E. (Ed.). . Constructing democracy: Human rights citizenship, and society in Latin America. Boulder: Westview press, 1996. p. 101-119.

LEFEBVRE, H. El derecho a la ciudad. 4. Ed. (1e ed. Barcelona: Editorial Penìnsula, 1969.

LEFEBVRE; HENRI. The production of space. 1974. ed. Oxford - Cambridge: Blackwell, 1997.

MATHIVET, C. propuesta de crear " Otra ciudad possible " Llamamiento. 2009.

MATHIVET, C. El derecho a la ciudad: claves para entender la propuesta de crear «Otra ciudad posible». Ciudades para tod@s Por el derecho a la ciudad, propuestas y experiencias, p. 23-28, 2010.

MINISTERIO DE AMBIENTE VIVIENDA Y DESARROLLO TERRITORIAL. Resolución 1687 (3.09.2009). . 2009, p. 69.

MIRAFTAB, F. Invited and Invented Spaces of Participation: Neoliberal Citizenship and Feminists' Expanded Notion of Politics. Wagadu, v. 1, p. 1-7, 2004.

MUÑOZ, F. Urbanalización: paisajes comunes, lugares globales. Barcelona: Gustavo Gili, 2008.

MURILLO, F. N. et al. Enseñar y aprender el derecho a la ciudad: El aporte de los talleres barriales de diagnostico socio-territorial a la socialización de los procesos de investigación. Urbanhabitat.Com.Ar, p. 1-16, 2010. 
NELSON, A. The praxis of sustainability citizenship. London - New York: Earthscan, 2016.

PIERCE, J.; MARTIN, D. G.; MURPHY, J. T. Relational place-making: the networked politics of place. Transactions of the Institute of British Geographers, n. 36, p. 54-70, 2011.

PONCE SOLÉ, J. Siete consideraciones sobre el derecho a la vivienda, la ciudad y las viviendas vacías: podemos hacer más (y mejor). Revista Catalana de Dret Públic. BLOG, p. 1-12, 2015.

RAWLS, J. Teoría de la justicia. 2da Edició ed. México: Fondo de Cultura Económica, 2006.

RODRÍGUEZ, A.; SUGRANYES, A. Vivienda privada de ciudad. Revista de Ingeniería, n. 35, p. 100-107, 2011.

ROLNIK, R.; DAPENA, L. Neoliberalismo reciente: la financialización de la vivienda propia y el derecho a la vivienda. Bitácora Urbano Territorial, p. 1-20, 2014.

SARTRE, J.-P. Being and nothingness: An essay on phenomenological ontology. London - New York: Routledge, 2008.

SCHÜTZ, E. J. Ciudades en América latina: Desarrollo barrial y vivienda. Santiago, Chile.: Ediciones SUR, 1996.

SOJA, E. W. The city and spatial justice. justice spatiale | spatial justice, n. 1, p. 1-5, 2009. 
SOTO VILLAGRÁN, P. El miedo de las a la violencia en la ciudad de México. Una cuestión de justicia espacial. Revista INVI, n. 27, p. 145-169, 2012.

SUGRANYES, A. El derecho a la ciudad: Praxis de la utopía. Habitat y sociedad, n. 1, p. 71-79, 2010.

SUGRANYES, A.; MATHIVET, C. Cities for All: Proposals and Experiences towards the Right to the City. Santiago: Habitat International Coalition, 2010.

TORRES TOVAR, C. A. Vivienda y hábitat en Colombia desde una perspectiva de derechos. Cuadernos Electrónicos, n. 6, p. 1-29, 2009.

TURNER, B. S. Four models of citizenship: From authoritarianism to consumer citizenship. En: Shifting Frontiers of Citizenship: The Latin American Experience. Amsterdaam: Brill, 2012. p. 55-82.

VIERA, E. Derecho a la ciudad-Herramienta de inclusión social en Latinoamércia. Revista Electrónica de Psicología Política, v. 28, p. 27-47, 2012.

WALS, A.; LENGLET, F. Sustainability citizenship: Collaborative and disruptive social learning. En: Sustainability Citizenship in Cities: Theory and practice. London - New York: Earthscan, 2016. p. 56-66. 